\title{
Reconstruction of the insulin-like signalling pathway of Haemonchus contortus
}

\author{
Namitha Mohandas ${ }^{1}$, Min Hu, Andreas J. Stroehlein ${ }^{1}$, Neil D. Young ${ }^{1}$, Paul W. Sternberg ${ }^{3}$, James B. Lok ${ }^{4}$ \\ and Robin B. Gasser ${ }^{1 *}$
}

\begin{abstract}
Background: In the present study, we reconstructed the insulin/insulin-like growth factor 1 signalling (IIS) pathway for Haemonchus contortus, which is one of the most important eukaryotic pathogens of livestock worldwide and is related to the free-living nematode Caenorhabditis elegans.

Methods: We curated full-length open-reading frames from assembled transcripts, defined the complement of genes that encode proteins involved in this pathway and then investigated the transcription profiles of these genes for all key developmental stages of $\mathrm{H}$. contortus.

Results: The core components of the IIS pathway are similar to their respective homologs in C. elegans. However, there is considerable variation in the numbers of isoforms between $\mathrm{H}$. contortus and C. elegans and an absence of AKT-2 and DDL-2 homologs from $\mathrm{H}$. contortus. Interestingly, DAF-16 has a single isoform in $\mathrm{H}$. contortus compared with 12 in C. elegans, suggesting novel functional roles in the parasitic nematode. Some IIS proteins, such as DAF-18 and SGK-1, vary in their functional domains, indicating distinct roles from their homologs in C. elegans.

Conclusions: This study paves the way for the further characterization of key signalling pathways in other socioeconomically important parasites and should help understand the complex mechanisms involved in developmental processes.
\end{abstract}

Keywords: Nematode, Haemonchus contortus, Insulin-like signalling pathway

\section{Background}

Roundworms (nematodes) are one of the most diverse groups of organisms on the planet. Some are free-living, and many are parasitic, causing substantial disease and socioeconomic problems globally. For example, Haemonchus contortus (the barber's pole worm; order Strongylida) is one of the most destructive parasitic nematodes of livestock animals (small ruminants, including sheep and goats) due to its high pathogenicity and widespread occurrence around the world [1]. This nematode feeds on blood from capillaries in the stomach (abomasum) wall, and causes haemorrhagic gastritis, anaemia, oedema and associated complications, often leading to the death of severely affected animals. $H$. contortus is transmitted orally from contaminated pasture to the host through a direct life cycle involving three free-living larval stages, of which the

\footnotetext{
*Correspondence: robinbg@unimelb.edu.au

${ }^{1}$ The University of Melbourne, Faculty of Veterinary and Agricultural Sciences, Parkville, VIC, Australia

Full list of author information is available at the end of the article
}

infective third larval stage (iL3) is ingested [2]. After a histotropic phase in the host animal, the larvae develop to the fourth stage (L4) and then to adults, which both feed on blood and cause pathogenic effects in the host animal.

The recent characterization of transcriptomes and draft genomes of $H$. contortus [3, 4] provides a solid basis for future studies of its developmental and reproductive biology using genetic, genomic, proteomic and metabolomic tools. However, a lack of tractable functional genomic tools for $H$. contortus and related parasitic nematodes, and an inability to maintain their complete life cycles in vitro, hampers functional investigations of genes and gene products in these nematodes (cf. [5-12]). This contrasts the situation for the free-living nematode, Caenorhabditis elegans, the best characterized metazoan organism, which can be readily maintained, and used to investigate fundamental processes and mechanisms, such as dauer formation [13].

Caenorhabditis elegans, which belongs to "clade V" [14], is relatively closely related to $H$. contortus. Published 
information $[7,8,15-17]$ indicates similarity in entry into and exit from the "dauer state" between $C$. elegans and strongylid nematodes [18]. This arrested state occurs in $C$. elegans when the nematode encounters harsh environmental conditions, such as starvation, crowding and/or a high temperature $[19,20]$. The dauer form can survive for several months and then resume development to reproductive adults when conditions improve [19]. Consistent with $C$. elegans, $H$. contortus and related nematodes have a similar third larval stage (L3), which is relatively resistant to unfavourable conditions and does not feed because it is encased by a cuticular sheath [2]. The "dauer hypothesis" [18] contends that the resumption of iL3 development in parasitic nematodes is functionally and developmentally analogous to the exit from dauer in C. elegans, and is regulated by similar mechanisms $[13,15,16,21]$.

Dauer development is governed by multiple signalling pathways, including the insulin/insulin-like growth factor 1 (IGF1)-like signalling (IIS) pathway [13], which, in $C$. elegans, comprises proteins such as DAF-2 (insulin-like receptor kinase [22-24]), AGE-1 (phosphoinositide-3 (PI3) kinase [25-27]) and DAF-16 (FOXO-class transcription factor [28-30]). In C. elegans, signalling via DAF-2 activates AKT-1/2 by phosphorylation which, in turn, negatively regulates DAF-16, which functions as a central mediator of multiple biological processes, such as growth, development, reproduction, longevity, age and stress resistance [31].

While much is known about the IIS pathway in $C$. elegans (reviewed in [31]), only a few studies have explored the functions of selected parts of this pathway in $H$. contortus [32-34], and no study has yet investigated its full composition in this parasitic nematode. Therefore, in the present study, we (i) curated the full-length open reading frames (ORFs) and defined the complement of genes that encode peptides/proteins involved in IIS, (ii) studied the interactions of these genes and (iii) examined their transcription profiles in all key developmental stages of $H$. contortus.

\section{Methods}

We employed data relating to a published draft genome as well as transcriptomes of all key developmental stages (egg, first- to fourth-stage larvae (L1, L2, L3 and L4) and adult) and both sexes (L4 and adult) of $H$. contortus (NCBI BioProject accession no. PRJNA205202; [4, 35]). This draft genome is $\sim 320 \mathrm{Mb}$ in size and has been predicted to encode 23,610 proteins [4].

\section{Identification of genomic scaffolds containing genes encoding IIS pathway components}

From the complete, assembled transcriptome representing all eight stages or sexes of $H$. contortus [4], we identified and extracted assembled transcripts based on their homology matches ( $E$-value cut-off: $10^{-5}$ ) to all genes encoding insulin/insulin-like growth factor 1 signalling (IIS) proteins in C. elegans [35]. Then, we identified genomic scaffolds containing regions of homology to known IIS genes by mapping ( $E$-value cut-off: $10^{-5}$ ) all assembled transcripts using BLAT [36]. We also used IIS genes from the $H$. contortus draft genome predicted previously using MAKER2 [4, 37]. Open reading frames (ORFs) of individual assembled transcripts were inferred using the program GeneMark-ES [38, 39]. Using the Integrative Genomics Viewer (IGV) [40, 41], we then visually integrated all of these data to obtain a consensus sequence for individual coding regions.

Identification of protein domains, families and subfamilies Identifying IIS protein genes encoded in the draft genome allowed us to then define the complete set of full-length transcripts. ORFs were verified and corresponding coding regions inferred from these full-length transcripts using ORF-finder [42]. Each predicted protein was characterized by its primary amino acid sequence and structural and/or functional domains, inferred using all databases (i.e., PROSITE, HAMAP, Pfam, PRINTS, ProDom, SMART, TIGRFAMs, PIRSF, SUPERFAMILY, CATHGene3D and PANTHER) within InterProScan v.5.14.53 [43, 44]. Individual predicted proteins were classified according to family and/or subfamily using information in the PANTHER database v.9.0 $[45,46]$. Following a comparison of those inferred from transcripts and genomic exons encoding IIS genes with the corresponding C. elegans homologs, we were able to infer the full complement of full-length transcripts and protein sequences.

\section{Analysis of differential transcription}

Each set of quality-filtered, paired-end RNA-seq reads for each individual developmental stage or sex of $H$. contortus was mapped to the final complement of fulllength IIS transcripts using Burrows-Wheeler Aligner (BWA) software [47]. For each stage/sex, the numbers of reads that mapped to individual transcripts were established using the SAM tools algorithm [48]. The resultant read counts per transcript per developmental stage were used as input data for DESeq2 and edgeR $[49,50]$. Differential transcription was calculated by pairwise comparison of all free-living (egg, L1, L2 and L3) and parasitic (L4 and adult) stages. Genes were recorded as differentially transcribed, using edgeR-calculated common and gene-wise dispersion factors, if the $\log _{2}$ fold change $\left(\log _{2}\right.$ FC) between free-living and parasitic stages compared with the normalised read count data was $\geq 2$, with a false discovery rate $(\mathrm{FDR})$ of $\leq 0.05$. A heat map (representing mapped reads) was produced using the heatmap.2 function in the gplots package in R [51]. 


\section{Results}

Identification and characterization of IIS signalling protein genes

From the complete, assembled transcriptome of $H$. contortus, we identified and extracted 3792 assembled transcripts based on their homology matches ( $E$-value cut-off: $10^{-5}$ ) to the 45 IIS protein genes, and then located the regions in genomic scaffolds to which these assembled transcripts mapped. The manual curation of the matching genomic and transcriptomic data for $H$. contortus identified 27 of 41 IIS gene homologs, and 4 of 40 insulin-like peptide (ILP) gene homologs using C. elegans genes as references.

Four, 18 and 9 of the 31 full-length transcripts encoded proteins involved in the upstream, conserved and downstream components of the IIS pathway, respectively. The features of these 31 predicted proteins (including lengths and pairwise sequence identities to their C. elegans homologs) are summarized in Table 1. Specifically, the numbers of isoforms of the predicted IIS genes varied from those of homologs encoded in C. elegans. Genes, such as the insulin-like peptide (ins-1), phosphoinositide 3-kinase (age-1/pi3k), heat-shock factor (hsf-1) and serine/threonine phosphatase (pptr-1), each have two or three isoforms in $H$. contortus, compared with only one molecule in C. elegans. In contrast, genes, such as those encoding the insulin receptor (daf-2), the phosphoinositidedependent kinase $(p d k-1)$, the 14-3-3 protein (par-5 and $f t t-2)$, the Nrf family transcription factor $(s k n-1)$ and the FoxO family transcription factor (daf-16), express single transcripts in $H$. contortus compared with 2 to 12 isoforms in $C$. elegans (Table 1). Individual predicted protein sequences ranged in length from 70 to 1455 amino acids, comparable with their corresponding C. elegans homologs, and these sequences shared $14.9 \%$ to $84.3 \%$ identity to their C. elegans homologs upon pairwise comparison (Table 1).

InterProScan analysis allowed the classification of predicted IIS proteins, based on domains and protein signatures (Additional file 1: Figure S1). The proteins predicted for $H$. contortus (i.e., Hc-PDK-1, -PPTR-1, -AKT-1, -DAF16, -HSB-1, -SKN-1 and -EGL-9) had the domains and signatures that were consistent with their respective $C$. elegans homologs. Hc-DAF-2 consisted of a protein kinase ATP-binding region signature (PS00107), an iron-sulfur binding domain (PS51379) and a furin-like cysteine-rich region (SM00261), which have been found in a number of eukaryotic proteins, such as epidermal growth factor receptor, endoprotease-4 and receptor tyrosine-protein kinase (LET-23), known to be involved in signal transduction by receptor tyrosine kinases [52-55]. Hc-DAF-18 lacked the protein tyrosine phosphatase catalytic domain (PTPc) motif and, instead, contained a dual-specificity phosphatase catalytic domain (PF00782). The pleckstrin homology (PH) domain (SM00233) was not present in Hc-PDK-1. HcPPTR-2 (protein phosphatase 2A) consisted of a unique leucine-rich repeat variant (G3DSA: 1.25.10.10) that represents a super-helical structure predicted to aid the binding of large substrates [56]. In comparison to the 14-3-3 proteins FTT-2 and PAR-5 of C. elegans, which have two protein signatures in PROSITE, the predicted $H$. contortus proteins have only protein signature 1 (PS00796). PANTHER family and subfamily classifications could not be assigned to some predicted IIS proteins of $H$. contortus, such as $H c$-INS-1, Hc-DDL- 1 and Hc-PAR-5. The regulatory subunit, Hc-IST-1, which contains the PH domain (SM00233; PS50003) belonging to the PH domain-like superfamily (SSF50729) is not present in $C$. elegans. The other adaptor protein, Hc-AAP-1, also contains the SH2 (Src homology 2) domain (PR00401), which is a 5-element fingerprint, and corresponds to the core structural element of the protein. The predicted $\mathrm{Hc}$-SGK-1 protein consists of the PX (phox) domain (PS50195; SSF64268), which is an important phosphoinositide-binding module with variable lipid-binding specificity $[57,58]$.

\section{Transcription profiles}

Significant differences in transcription were recorded among eight stages/sexes of $H$. contortus for some of the 31 genes involved in the IIS pathway (Fig. 1). The insulin-like peptide genes Hc-ins-1a, -ins-1b, -ins-17 and -ins-18 were highly transcribed in L1 to L3 stages of $H$. contortus, and significantly down-regulated in parasitic stages, particularly female L4 and adult stages. In contrast, downstream genes, such as Hc-daf-2, -aap-1, -ist-1, -age-1a, -age-1b, -daf-18, -pdk-1 and -akt-1, which encode IIS cytoplasmic signalling proteins, had the highest transcription in eggs, L3s and female adults (Fig. 1), except for Hc-sgk-1, which had limited transcription in both egg and L3 stages in comparison to all other stages. The 14-3-3 homologous genes, Hc-ftt-2 and Hcpar-5, known to regulate daf-16 in C. elegans $[59,60]$ were highly transcribed in all life cycle stages, while Hc-daf-16 was highly transcribed only in the egg, L2 and L3 stages (Fig. 1). Downstream genes transcriptionally regulated by $d a f-16(s k n-1, h s f-1, h s b-1, d d l-1$ and egl-9) were transcribed at high levels in eggs, L3s and in females of both haematophagous (i.e., L4 and adult) stages (Fig. 1).

\section{The IIS signalling pathway of $\boldsymbol{H}$. contortus}

Of a total of 81 genes involved in the IIS pathway of $C$. elegans [31], 31 homologs were identified in $H$. contortus. Specifically, in $H$. contortus, four insulin-like peptide (ILP) homologs of 40 in C. elegans as well as 9 of 11 downstream components of the IIS pathway were identified. However, C. elegans akt-2 and $d d l-2$ homologs were 
Table 1 Pairwise comparisons of sequence identity (\%) of proteins representing the insulin/insulin-like growth factor 1(IGF1)-like signalling pathway (IIS) between Haemonchus contortus and Caenorhabditis elegans

\begin{tabular}{|c|c|c|c|c|c|c|}
\hline Protein & Scaffold & Transcripts representing the IIS genes & Length (aa) & $\begin{array}{l}\text { Homologs in } \\
\text { C. elegans (Gene code) }\end{array}$ & Length (aa) & $\begin{array}{l}\text { Pairwise sequence } \\
\text { identity (\%) }\end{array}$ \\
\hline Hc-INS-1a & scaffold1633 & Locus_6343_Transcript_1/2_Confidence_0.667_Length_624 & 100 & F13B12.5 & 109 & 42.0 \\
\hline Hc-INS-1b & scaffold11919 & Locus_3137_Transcript_1/2_Confidence_0.750_Length_686 & 100 & F13B12.5 & 109 & 42.0 \\
\hline HC-INS-17 & - & Locus_4104_Transcript_1/1_Confidence_1.000_Length_511 & 106 & F56F3.6 & 108 & 54.7 \\
\hline HC-INS-18 & & Locus_1267_Transcript_2/3_Confidence_0.600_Length_720 & 70 & $\mathrm{~T} 28 \mathrm{~B} 8.2$ & 95 & 47.4 \\
\hline HC-DAF-2 & scaffold 13413 & Locus_7014_Transcript_2/3_Confidence_0.714_Length_5695 & 1455 & $\begin{array}{l}\text { Y55D5A.5a, b, c*, } \\
\text { d, e, f, g }\end{array}$ & $672-1928$ & $25.5-34.6$ \\
\hline HC-IST-1 & scaffold434 & $\begin{array}{l}\text { Locus_5794_Transcript_4/8_Confidence_0.650_Length_4263 + } \\
\text { Locus_5794_Transcript_6/8_Confidence_0.500_Length_3032 }\end{array}$ & 1441 & C54D1.3 & 1003 & 18.4 \\
\hline HC-AAP-1 & scaffold6618 & Locus_7479_Transcript_1/1_Confidence_1.000_Length_1541 & 427 & Y110A7A.10 & 522 & 36.1 \\
\hline HC-AGE-1a & C279481 & Locus_4139_Transcript_1/1_Confidence_1.000_Length_3713 & 1156 & B0334.8a & 1182 & 40.5 \\
\hline HC-AGE-1b & scaffold1062 & Locus_10568_Transcript_2/2_Confidence_0.800_Length_3699 & 1149 & B0334.8a & 1182 & 40.6 \\
\hline HC-DAF-18 & & Locus_6617_Transcript_3/3_Confidence_0.714_Length_3487 & 800 & T07A9.6 & 962 & 24.0 \\
\hline HC-PDK-1 & C457723 & Locus_2599_Transcript_2/3_Confidence_0.500_Length_2764 & 576 & $\mathrm{H} 42 \mathrm{~K} 12.1 \mathrm{a}, \mathrm{b}^{*}$ & $632-636$ & $41.8-42.4$ \\
\hline Hc-SGK-1a & - & Locus_1010_Transcript_1/1_Confidence_1.000_Length_1434 & 462 & W10G6.2a*, b & 453-463 & $60.0-61.2$ \\
\hline Hc-SGK-1b & - & Locus_5956_Transcript_1/1_Confidence_1.000_Length_1384 & 428 & W10G6.2a*, b* & 453-463 & 63.1 \\
\hline HC-AKT-1a & scaffold 15637 & Locus_6593_Transcript_2/2_Confidence_0.857_Length_2281 & 540 & C12D8.10a*, b, c & $254-546$ & $73.2-75.9$ \\
\hline HC-AKT-1b & scaffold 17120 & Locus_44_Transcript_10/11_Confidence_0.651_Length_6534 & 552 & C12D8.10a, b*, c & $254-546$ & $73.2-77.6$ \\
\hline Hc-PPTR-1a & scaffold4564 & Locus_1388_Transcript_2/4_Confidence_0.700_Length_4420 & 542 & W08G11 & 542 & 80.1 \\
\hline Hc-PPTR-1b & C217263 & Locus_3418_Transcript_2/2_Confidence_0.667_Length_3679 & 542 & W08G11 & 542 & 80.1 \\
\hline HC-PPTR-1C & C426137 & Locus_351_Transcript_5/7_Confidence_0.556_Length_5020 & 542 & W08G11 & 542 & 80.1 \\
\hline HC-PPTR-2 & scaffold6183 & Locus_8402_Transcript_1/1_Confidence_1.000_Length_3588 & 476 & $\mathrm{C} 13 \mathrm{G} 3.3 \mathrm{a}^{*}, \mathrm{~b}, \mathrm{c}^{*}, \mathrm{~d}^{*}$ & $557-607$ & $70.1-71.2$ \\
\hline HC-FTT-2 & - & Locus_4342_Transcript_1/6_Confidence_0.556_Length_1420 & 206 & F52D10.3a, b* & $198-248$ & $80.5-84.3$ \\
\hline HC-PAR-5 & - & $\begin{array}{l}\text { Locus_4342_Transcript_5/6_Confidence_0.667_Length_1898+ } \\
\text { Locus_4342_Transcript_3/6_Confidence_0.667_Length_1669 }\end{array}$ & 201 & $M 117.2 a^{*}, b$ & $126-248$ & $48.4-82.6$ \\
\hline Hc-DAF-16 & C472265 & Locus_5600_Transcript_3/3_Confidence_0.714_Length_3611 & 589 & $\begin{array}{l}\text { R13H8.1a, b, c, d, e, f, } \\
g, h, i, k, I, m^{*}\end{array}$ & $303-589$ & $32.4-43.2$ \\
\hline HC-SKN-1 & scaffold 10742 & Locus_3183_Transcript_5/6_Confidence_0.684_Length_3607 & 612 & T19E7.2a, b, c, d* & $223-623$ & $30.3-38.5$ \\
\hline HC-HSF-1a & scaffold1518 & Locus_7749_Transcript_2/2_Confidence_0.875_Length_2372 & 544 & Y53C10A.12 & 671 & 33.5 \\
\hline Hc-HSF-1b & scaffold3772 & Locus_497_Transcript_5/5_Confidence_0.577_Length_2356 & 544 & Y53C10A.12 & 671 & 33.5 \\
\hline HC-HSB-1 & scaffold9500 & Locus_3235_Transcript_1/1_Confidence_1.000_Length_453 & 91 & K08E7.2 & 80 & 60.0 \\
\hline HC-DDL-1 & scaffold 11860 & $\begin{array}{l}\text { Locus_4675_Transcript_1/2_Confidence_0.750_Length_767 + } \\
\text { Locus_8707_Transcript_2/3_Confidence_0.667_Length_738 }\end{array}$ & 129 & F59E12.10 & 189 & 27.1 \\
\hline Hc-EGL-9a & scaffold12571 & Locus_5190_Transcript_1/1_Confidence_1.000_Length_1701 & 504 & $\begin{array}{l}F 22 E 12.4 a, b, c \\
d^{*}, e\end{array}$ & $363-723$ & 14.9-48.6 \\
\hline Hc-EGL-9b & C449377 & Locus_3254_Transcript_2/2_Confidence_0.333_Length_2254 & 482 & $F 22 E 12.4 a, b, c, d^{*}, e$ & $363-723$ & $14.9-51.2$ \\
\hline Hc-EGL-9c & scaffold 12558 & Locus_1766_Transcript_1/1_Confidence_1.000_Length_2016 & 482 & $\begin{array}{l}\text { F22E12.4a, b, c*, } \\
\text { d, e }\end{array}$ & $363-723$ & $14.9-49.5$ \\
\hline Hc-EGL-9d & C457287 & Locus_4349_Transcript_1/1_Confidence_1.000_Length_1791 & 482 & $F 22 E 12.4 a, b, c^{*}, d, e$ & $363-723$ & $14.9-49.5$ \\
\hline
\end{tabular}




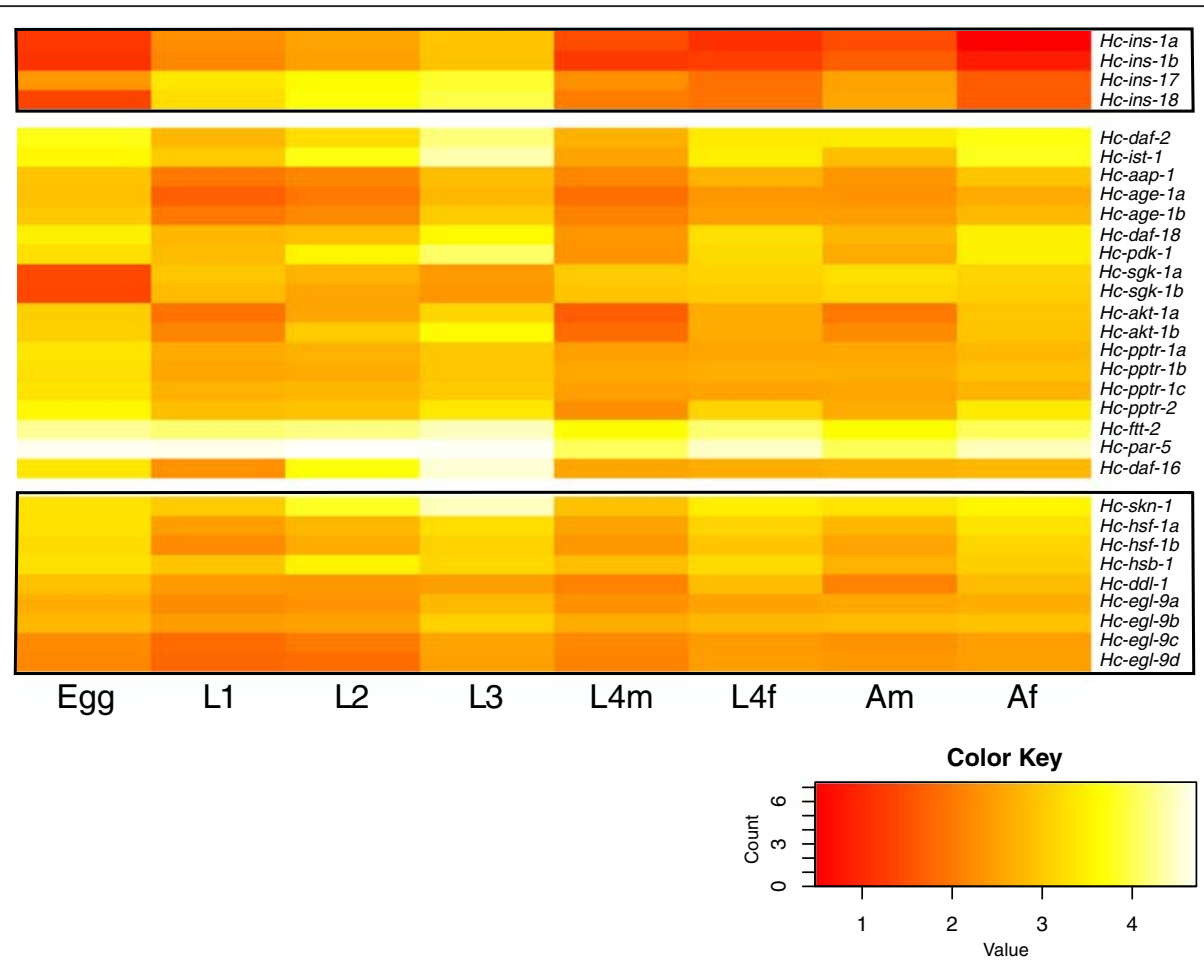

Fig. 1 Heat-map displaying transcription profiles for genes (cf. Table 1) involved in insulin/insulin-like growth factor 1 (IGF1)-like signalling (IIS) in Haemonchus contortus. Hc-ins-1a/1b, -ins-17 and -ins-18 are genes that act upstream (U) of the IIS pathway. Genes Hc-daf-2, -ist-1, -aap-1, -age-1a/16, - daf-18, -pdk-1, -sgk-1a/1b, - akt-1a/1b, -pptr-1a/1b/1c, -pptr-2, -ftt-2, -par-5 and Hc-daf-16 are part of the core (C) IIS pathway and genes Hc-skn-1, $-h s f-1 a / 1 b,-h s b-1,-d d l-1$ and $H c-e g l-9 a / b / c / d$ are activated downstream (D) of the IIS pathway. Transcription levels in different developmental stages (egg, L1, L2, L3, L4 and A = adult; $f$ = female; $m$ = male) of H. contortus (see colour scale): low (red), medium (orange), high (yellow) and very high (white)

not found in $H$. contortus, which was confirmed by searching independent genomic and transcriptomic data sets [3]. Therefore, overall, the number of core IIS genes (Hc-daf-2, -ist-1, -aap-1, -age-1, -daf-18, -pdk-1, -sgk-1, -akt-1, -pptr-1, -ftt-2, -par-5 and -daf-16) was similar, with variation mainly in the numbers of isoforms between $H$. contortus and C. elegans.

Using information available for C. elegans (from WormBase; [35]), we constructed the IIS pathway for the genes predicted in $H$. contortus (Fig. 2). In this pathway, insulinlike peptides encoded by Hc-ins-1a, -ins-1b, -ins-17 and -ins-18 are predicted to interact with DAF-2 insulin receptor, thereby activating the receptor. This, in turn, should facilitate the recruitment of regulatory proteins, $H c$-IST-1 and -AAP-1, to activate phosphoinositide 3-kinase ( $\mathrm{Hc}$ AGE-1). The activation of Hc-AGE-1 likely results in a conversion of phosphatidylinositol 4,5-bisphosphate $\left(\mathrm{PIP}_{2}\right)$ to phosphatidylinositol 4,5-trisphosphate $\left(\mathrm{PIP}_{3}\right)$, which, in turn, activates phosphoinositide-dependent protein kinase1 (-PDK-1). A regulatory lipid phosphatase, $H c$-DAF-18, is inferred to act as an antagonist to -AGE-1 activity by dephosphorylating $\mathrm{PIP}_{3}$. Downstream serine/threonine kinases, $H c$-AKT-1 and $H c$-SGK-1, are predicted to be activated by $H c$-PDK-1, resulting in the phosphorylation of $H c$-DAF-16 (FoxO transcription factor). The protein phosphatases $H c$-PPTR-1 and -PPTR-2 act as regulatory subunits that bind and dephosphorylate $\mathrm{Hc}$ AKT-1. The phosphorylated Hc-DAF-16 interacts with the regulatory proteins $H c$-FTT-2 and Hc-PAR-5, inhibiting its subcellular localization into the nucleus and subsequent regulation of DAF-16 target genes. A number of downstream target genes are involved in various processes, such as stress resistance, dauer formation, the regulation of life span and the activation of transforming growth factor beta (TGF- $\beta$ ) signalling. The regulation of IIS genes is important, as it affects the transcription of downstream gene targets that may result in loss-offunction phenotypes.

\section{Discussion}

Using a bioinformatic approach, we reconstructed the IIS pathway of Haemonchus contortus from transcriptomic and genomic data sets for this nematode, and explored the transcription of individual genes as well as their interactions. The composition of the core IIS pathway in $H$. contortus was relatively consistent with that of C. elegans, although there were considerable differences between these nematodes in the upstream and downstream targets of this pathway. In the present study, four insulin-like peptides (ILPs) were identified in $H$. contortus, as opposed to 


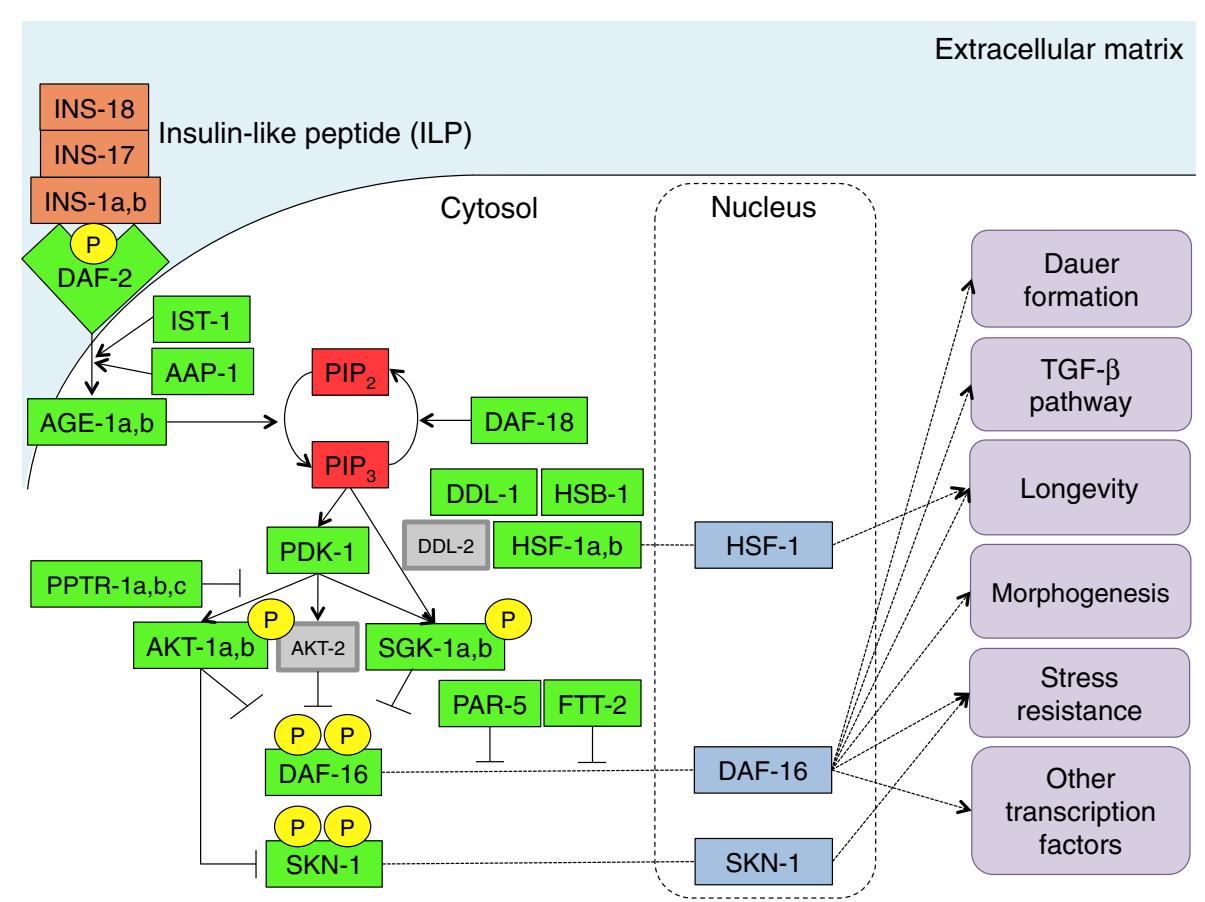

Fig. 2 Reconstruction of the insulin/insulin-like growth factor 1 (IGF1)-like signalling (IIS) pathway of Haemonchus contortus. Four insulin-like peptides (ILPS) are encoded in H. contortus (orange) compared with 40 in Caenorhabditis elegans. All core functional gene products (green) of the IIS pathway, except for AKT-2 and DDL-2 (grey), are encoded in H. contortus. Additional transcription factors activated downstream of the IIS pathway, such as SKN-1 and HSF-1, are also predicted in H. contortus. Phosphatidylinositol $(3,4,5)$-trisphosphate $\left(\mathrm{PIP}_{3}\right)$ is the product of the class I phosphoinostide 3-kinases (PI 3-kinases) phosphorylation of phosphatidylinositol (4,5)-bisphosphate $\left(\mathrm{PIP}_{2}\right)$; it is a phospholipid in the plasma membrane. Phosphorylation represented by $\mathrm{P}$ (yellow). The insulin pathway controls the transcription of various genes, and regulates key processes, including dauer formation, longevity, stress resistance and morphogenesis, as well as other pathways such as TGF- $\beta$ and involving other key transcription factors

40 ILPs found in C. elegans [61-64]. A similar, marked reduction in the repertoire of ILP-encoding transcripts has been observed in the parasitic nematode Strongyloides stercoralis (clade IV), where only seven of the 40 ILPs of C. elegans are represented [65]. The four ILPs predicted for $H$. contortus all have representative functional domains and protein signatures, including an insulin-like domain representing the insulin/IGF/relaxin family, a disulphiderich alpha-helical domain and an insulin family signature (cf. Additional file 1: Figure S1). In C. elegans, several ILPs (encoded by ins-1, ins-6, ins-7) have been shown to regulate dauer formation, longevity and development [31]. Although the functions of all 40 ILPs are not yet known, some (e.g., encoded by ins-1, ins-6, ins-7 and daf-28) have been extensively studied [63, 64, 66-68]. An interesting feature of these peptides is that they can either act as agonists or antagonists of DAF-2, the only insulin-like receptor kinase in C. elegans [63, 66, 67, 69]. While the basis of the differences in function is presently unknown, it is hypothesized that neural inputs from chemosensory neurons in the amphids and transduced by $G$ protein-coupled receptors (GPCRs) therein trigger specific profiles of ILP expression, possibly including optimum levels of agonists and antagonists in one or a few developmental stages, which combine to precisely regulate the insulin signalling pathway response (i.e., downstream gene expression) to the complex set of environmental cues experienced by this free-living nematode [70]. It is likely that ILP profiles are similarly regulated in parasitic nematodes. Indeed, the regulation of ILP expression by upstream cyclic GMP signalling has been observed in $S$. stercoralis, where the administration of 8-bromo-cGMP to cultured larvae elicits naturally occurring profiles of ILP expression, in contrast to the baseline levels of expression observed in untreated controls [71]. The marked reduction in numbers of ILPs in parasitic nematodes examined to date might reflect a rather more specific interaction with their environments relative to more opportunistic free-living organisms, such as C. elegans. The homologs of the $C$. elegans ILPs identified in $H$. contortus were encoded by Hc-ins-1a, -ins-1b, -ins-17 and -ins-18, which all represent antagonists, implying that the regulation of the IIS pathway is dependent on their expression patterns in all life stages of the parasite. High transcription in the L1, L2 and L3 stages of $H$. contortus and considerably lower transcription in L4 and adult stages indicate that the ILPs of $H$. contortus are transcriptionally regulated during the transition from free-living to parasitic stages. 
By contrast to $H$. contortus, the seven ILPs of $S$. stercoralis constitute a set of peptides whose structures and patterns of expression in free-living and parasitic stages suggest members that are either agonists or antagonists of Ss-DAF-2. This apparent diversity of ILP function in $S$. stercoralis could explain the capability of the parasite to undertake either direct development to infective L3s or development to a generation of free-living male and female worms with many biological attributes in common with non-parasitic nematodes.

Some of the intracellular protein components of the IIS pathway showed variation in the functional domains present and in the number of isoforms predicted. For instance, Hc-DAF-18 is a homolog of the mammalian PTEN protein, which is a well-recognised phosphatase and a tumour suppressor [72]. In C. elegans, DAF-18, a lipid phosphatase, acts by inhibiting the activation of PDK-1 by dephosphorylating phosphatidylinositol $(3,4,5)$-trisphosphate $\left(\mathrm{PIP}_{3}\right)$ to phosphatidylinositol 4,5bisphosphate $\left(\mathrm{PIP}_{2}\right)$ by removing the phosphate in the D3 position of the inositol ring [73]. Ce-DAF-18 is also suggested to have tyrosine phosphatase activity, based on the presence of its functional catalytic domain. However, in $H$. contortus, while $H c$-DAF-18 might have the same lipid phosphatase function as its C. elegans homo$\log$, the presence of a dual-specificity phosphatase catalytic domain indicates its ability to dephosphorylate both tyrosine- and serine-/threonine-phosphorylated proteins. This suggested, additional function of $\mathrm{Hc}_{c}$-DAF-18 might hint to a functional role of the signalling pathway in recovery from developmental arrest.

The C. elegans insulin-signalling pathway activates two Akt family members, AKT-1 and AKT-2, as well as a serum and glucocorticoid-inducible kinase, SGK-1 downstream of the phosphoinositide-dependent kinase, PDK-1. All of these serine-threonine kinases are activated by AGE-1/PI3K. Previous studies [74-76] have shown that null-mutants of Ce-akt-1 and Ce-akt-2 result in nonconditional dauer arrest and an extension of lifespan. A knockdown of $s g k-1$ by RNAi also induces an extended lifespan, indicating that it functions in a similar manner to AKT-1 and AKT-2 [77]. Interestingly, in H. contortus, only two kinases were predicted downstream of $H c$-PDK-1, namely $H c$-AKT-1 and $H c$-SGK-1. The absence of AKT-2 might indicate a distinct regulation of IIS pathway in this nematode during the switch to the parasitic stage and also during reproduction. The transcription of Hc-akt-1 was higher in females than in males. The assessment of the functional domains of $\mathrm{Hc}$-SGK-1 revealed the presence of a PX (phox) domain, which was not present in its respective C. elegans homolog. PX, a phospholipidbinding domain, primarily interacts with $\mathrm{PIP}_{3}$ lipids [78]. The presence of this unique domain is likely to compensate for the deficiency of AKT-2 by allowing an activation of $H c$-SGK-1 by both $H c$-PDK-1 as well as $\mathrm{PIP}_{3}$, thereby effecting the expression of downstream Hc-DAF-16 target genes.

DAF-16 is a member of the FoxO family of forkhead transcription factors, which are regulators of growth, metabolism, stress response, cell cycle control and longevity in many organisms [79]. The nuclear translocation of DAF-16 from the cytoplasm is inhibited by the phosphorylation at its RxRxxS/T motifs [80, 81], which are conserved among C. elegans DAF-16, mammalian FoxOs and predicted $H$. contortus daf-16. The $C$. elegans genome encodes twelve DAF-16 transcripts. Although the functions of these isoforms are not known, it is hypothesized that they have distinct tissue distributions in hypodermis, muscle, neurons, and intestine [29, 80-83]. Studies of $\mathrm{Ce}$-DAF-16 indicate that biological functions of the isoforms vary according to their tissue distribution [80, 83]. In contrast, in $H$. contortus, a single homolog of DAF-16 was predicted. This finding implies that the array of target genes predicted to be transcriptionally regulated by $\mathrm{Hc}$ DAF-16 is controlled by a single homolog, possibly resulting in a more complex regulation of downstream gene targets and distinct phenotypes. Given that the input signals of IIS pathway are also lesser in number compared with $C$. elegans, this information suggests a novel regulatory mechanism that differs between freeliving and parasitic nematodes. This hypothesis is bolstered by a similar reduction in transcripts from the daf-16 ortholog in S. stercoralis. Here, there are only two transcripts, designated $S s-d a f-16$ a and $S s-d a f-16 \mathrm{~b}$, each expressed under the control of a different promoter $[65,84]$.

The 'dauer hypothesis' suggests that a similar mechanism of action takes place in major signalling pathways including the IIS, cyclic GMP and TGF- $\beta$ pathways, which control the entry into and exit from arrested development in C. elegans, as in parasitic nematodes [18]. The present investigation of genomic and transcriptomic data sets from $H$. contortus suggests that the major intracellular signalling components of IIS, such as daf-2, age-1, pdk-1 and akt-1, likely have similar functions to C. elegans homologs. This hypothesis has been supported for age-1 orthologs in other parasitic nematodes by the fact that the PI3K inhibitor LY294002 suppresses developmental activation of iL3 under host-like culture conditions [85-87]. However, the roles of key genes encoding ILPs, DAF-18, SGK-1 and DAF-16 in H. contortus appear to be distinct.

Although the 'dauer hypothesis' usually considers L3 arrest in parasitic nematodes to be analogous to dauer in C. elegans [18], H. contortus and related nematodes, such as Ostertagia and Cooperia spp., can undergo hypobiosis at the early L4 stage within the host animal [88]. Given that this latter adaptive state enables transitional parasite 
survival within the host, and regulates parasite transmission and population size [88], understanding this phenomenon is of critical importance. Hence, future work should evaluate the involvement of IIS and associated signalling pathways in hypobiosis. It would be of particular interest to reconstruct the TGF- $\beta$ signalling pathway in $H$. contortus and other trichostrongylids, and assess transcription profiles throughout development, given the major contrast in transcription at the L3 stage between various parasitic nematodes including $H$. contortus (up-regulation) and C. elegans (down-regulation) (cf. [18]) that suggests an altogether unique function of DAF-7 in parasitic worms. This line of investigation will be interesting in light of the fact that $C$. elegans offers no exact counterpart to the early L4 arrest that occurs in these economically important trichostrongyles. As such, mechanisms of early L4 arrest will likely represent a unique adaptation to parasitism for some clade $\mathrm{V}$ nematodes.

\section{Conclusions}

In conclusion, the availability of transcriptomic and draft genomic data sets for $H$. contortus has enabled the first detailed bioinformatic exploration of the IIS pathway in this parasite. We curated the full-length transcripts and defined the complement of genes that encode peptides/ proteins involved in this pathway by comparison with $C$. elegans, reconstructed the pathway with these genes and investigated their transcription profiles in key developmental stages of $H$. contortus. We hope that reconstructing the IIS pathway for $H$. contortus will provide a stepping stone for future studies of development, reproduction, ageing, longevity, metabolism and/or behaviour in this important parasitic worm, and a stimulus to explore other signalling pathways in socioeconomically important strongylids.

\section{Additional file}

Additional file 1: Figure S1. A schematic representation of functional domains and motifs as predicted by InterProScan of members of the insulin/ insulin-like growth factor 1 (IGF1)-like signalling (IIS) pathway of Haemonchus contortus inferred from full-length transcripts and their Caenorhabditis elegans homologs. (PDF $216 \mathrm{~kb}$ )

\section{Competing interests}

The authors declare that they have no competing interests.

\section{Authors' contributions}

Conceived and designed the study and supervised the project: RBG. Undertook the study and data analysis: NM and RBG. Contributed to developing bioinformatic tools: NM, NDY and RBG. Contributed to the interpretation of findings, and drafting or formatting of the manuscript: $N M, R B G, J B L, M H$, AJS and PWS. Planned and wrote the paper: NM, RBG, JBL and MH. All authors read and approved the final version of the manuscript.

\section{Acknowledgements}

Funding from the Australian Research Council (ARC) and the National Health and Medical Research Council of Australia (NHMRC) is gratefully acknowledged, as is support from the Victorian Life Sciences Computation Initiative VLSCl; grant number VR0007) (RBG). Other support was from grants from the National Key Basic Research Program (973 program) of China (no. 2015CB150300), the National Natural Science Foundation of China (NSFC) (no. 31172310) (MH) and from the National Institute of Health (NIH), USA (Al-50688) (JBL). We acknowledge the contributions of staff at WormBase (www.wormbase.org). AJS is a recipient of a Melbourne International Research Scholarship (MIRS) and a Melbourne International Fee Remission Scholarship (MIFRS) from The University of Melbourne.

\section{Author details}

${ }^{1}$ The University of Melbourne, Faculty of Veterinary and Agricultural Sciences, Parkville, VIC, Australia. ${ }^{2}$ State Key Laboratory of Agricultural Microbiology, College of Veterinary Medicine, Huazhong Agricultural University, Wuhan 430070 Hubei, China. ${ }^{3} \mathrm{HHMl}$, Division of Biology, California Institute of Technology, Pasadena, CA, USA. ${ }^{4}$ Department of Pathobiology, School of Veterinary Medicine, University of Pennsylvania, 3800 Spruce Street, Philadelphia, PA 19104, USA.

\section{Received: 12 January 2016 Accepted: 26 January 2016}

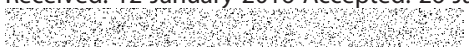

\section{References}

1. Gasser RB, von Samson-Himmelstjerna G. Haemonchus contortus and Haemonchosis Past, Present and Future Trends. Adv Parasitol. in press.

2. Veglia F. The anatomy and life-history of the Haemonchus contortus. Vet Res. 1915:4:347-500.

3. Laing R, Kikuchi T, Martinelli A, Tsai IJ, Beech RN, Redman E, et al. The genome and transcriptome of Haemonchus contortus, a key model parasite for drug and vaccine discovery. Genome Biol. 2013;14:R88.

4. Schwarz EM, Korhonen PK, Campbell BE, Young ND, Jex AR, Jabbar A, et al. The genome and developmental transcriptome of the strongylid nematode Haemonchus contortus. Genome Biol. 2013;14:R89.

5. Boyle JP, Yoshino TP. Gene manipulation in parasitic helminths. Int J Parasitol. 2003;33:1259-68.

6. Aboobaker AA, Blaxter ML. Functional genomics for parasitic nematodes and platyhelminths. Trends Parasitol. 2004;20:178-84.

7. Gilleard JS. The use of Caenorhabditis elegans in parasitic nematode research. Parasitology. 2004;128 Suppl 1:S49-70.

8. Britton C, Murray L. Using Caenorhabditis elegans for functional analysis of genes of parasitic nematodes. Int J Parasitol. 2006;36:651-9.

9. Kalinna BH, Brindley PJ. Manipulating the manipulators: advances in parasitic helminth transgenesis and RNAi. Trends Parasitol. 2007;23:197-204.

10. Knox DP, Geldhof P, Visser A, Britton C. RNA interference in parasitic nematodes of animals: a reality check? Trends Parasitol. 2007;23:105-7

11. Maule AG, McVeigh P, Dalzell JJ, Atkinson L, Mousley A, Marks NJ. An eye on RNAi in nematode parasites. Trends Parasitol. 2011;27:505-13.

12. Lok JB. Nucleic acid transfection and transgenesis in parasitic nematodes. Parasitology. 2012;139:574-88.

13. Hu PJ. Dauer. WormBook, ed. The C. elegans Research Community, WormBook. 2007. doi/10.1895/wormbook.1.144.1.

14. Blaxter ML, De Ley P, Garey JR, Liu LX, Scheldeman P, Vierstraete A, et al. A molecular evolutionary framework for the phylum Nematoda. Nature. 1998;392:71-5.

15. Hotez P, Hawdon J, Schad GA. Hookworm larval infectivity, arrest and amphiparatenesis: the Caenorhabditis elegans Daf-c paradigm. Parasitol Today. 1993;9:23-6.

16. Bürglin TR, Lobos E, Blaxter ML. Caenorhabditis elegans as a model for parasitic nematodes. Int J Parasitol. 1998;28:395-411.

17. Hashmi S, Tawe W, Lustigman S. Caenorhabditis elegans and the study of gene function in parasites. Trends Parasitol. 2001;17:387-93.

18. Crook M. The dauer hypothesis and the evolution of parasitism: 20 years on and still going strong. Int J Parasitol. 2014:44:1-8.

19. Golden JW, Riddle DL. The Caenorhabditis elegans dauer larva: developmental effects of pheromone, food, and temperature. Dev Biol. 1984;102:368-78.

20. Riddle DL, Albert PS. Genetic and environmental regulation of dauer larva development. In: Riddle DL, Blumenthal T, Meyer BJ, Priess JR, editors. C. elegans II. Cold Spring Harbor: Cold Spring Harbor Laboratory Press: 1997. ch. 26.

21. Blaxter M. Caenorhabditis elegans is a nematode. Science. 1998;282:2041-6. 
22. Kimura KD, Tissenbaum HA, Liu Y, Ruvkun G. daf-2, an insulin receptor-like gene that regulates longevity and diapause in Caenorhabditis elegans. Science. 1997;277:942-6.

23. Apfeld J, Kenyon C. Cell nonautonomy of C. elegans daf-2 function in the regulation of diapause and life span. Cell. 1998;95:199-210.

24. Gems D, Sutton AJ, Sundermeyer ML, Albert PS, King KV, Edgley ML, et al. Two pleiotropic classes of daf-2 mutation affect larval arrest, adult behavior, reproduction and longevity in Caenorhabditis elegans. Genetics. 1998;150:129-55.

25. Vanhaesebroeck B, Leevers SJ, Panayotou G, Waterfield MD. Phosphoinositide 3-kinases: a conserved family of signal transducers. Trends Biochem Sci. 1997;22:267-72.

26. Engelman JA, Luo J, Cantley LC. The evolution of phosphatidylinositol 3-kinases as regulators of growth and metabolism. Nat Rev Genet. 2006;7:606-19.

27. Ayyadevara S, Tazearslan C, Bharill P, Alla R, Siegel E, Shmookler Reis RJ. Caenorhabditis elegans PI3K mutants reveal novel genes underlying exceptional stress resistance and lifespan. Aging Cell. 2009;8:706-25.

28. Lin K, Dorman JB, Rodan A, Kenyon C. daf-16: An HNF-3/forkhead family member that can function to double the life-span of Caenorhabditis elegans. Science. 1997;278:1319-22.

29. Ogg S, Paradis S, Gottlieb S, Patterson Gl, Lee L, Tissenbaum HA, et al. The Fork head transcription factor DAF-16 transduces insulin-like metabolic and longevity signals in C. elegans. Nature. 1997;389:994-9.

30. Ogg S, G R. The C. elegans PTEN homolog, DAF-18, acts in the insulin receptorlike metabolic signaling pathway. Mol Cell. 1998;2:887-93.

31. Murphy CT, Hu PJ. Insulin/insulin-like growth factor signaling in C. elegans. WormBook, ed. The C. elegans Research Community, WormBook. 2013. doi/ 10.1895/wormbook.1.164.1.

32. Hu M, Lok JB, Ranjit N, Massey Jr HC, Sternberg PW, Gasser RB. Structural and functional characterisation of the fork head transcription factor-encoding gene, Hc-daf-16, from the parasitic nematode Haemonchus contortus (Strongylida). Int J Parasitol. 2010;40:405-15.

33. Li F, Lok JB, Gasser RB, Korhonen PK, Sandeman MR, Shi D, et al. Hc-daf-2 encodes an insulin-like receptor kinase in the barber's pole worm, Haemonchus contortus, and restores partial dauer regulation. Int J Parasitol. 2014;44:485-96.

34. Li FC, Gasser RB, Lok JB, Korhonen PK, Wang YF, Yin FY, et al. Exploring the role of two interacting phosphoinositide 3-kinases of Haemonchus contortus. Parasit Vectors. 2014;7:498.

35. https://www.wormbase.org/ (2016). Accessed 6 Jan 2016.

36. Kent WJ. BLAT - the BLAST-like alignment tool. Genome Res. 2002;12:656-64.

37. Holt C, Yandell M. MAKER2: an annotation pipeline and genome-database management tool for second-generation genome projects. BMC Bioinformatics. 2011;2:491.

38. Lomsadze A, Ter-Hovhannisyan V, Chernoff YO, Borodovsky M. Gene identification in novel eukaryotic genomes by self-training algorithm. Nucleic Acids Res. 2005;33:6494-506.

39. Ter-Hovhannisyan V, Lomsadze A, Chernoff YO, Borodovsky M. Gene prediction in novel fungal genomes using an ab initio algorithm with unsupervised training. Genome Res. 2008;18:1979-90.

40. Robinson JT, Thorvaldsdóttir H, Winckler W, Guttman M, Lander ES, Getz G et al. Integrative Genomics Viewer. Nature Biotechnol. 2011;29:24-6.

41. Thorvaldsdóttir H, Robinson JT, Mesirov JP. Integrative Genomics Viewer (IGV): high-performance genomics data visualization and exploration. Brief Bioinform. 2013;14:178-92.

42. http://www.ncbi.nlm.nih.gov/projects/gorf/ (2016). Accessed 6 Jan 2016

43. Zdobnov EM, Apweiler R. InterProScan - an integration platform for the signature-recognition methods in InterPro. Bioinformatics. 2001;17:847-8.

44. Jones P, Binns D, Chang HY, Fraser M, Li W, McAnulla C, et al. InterProScan 5: genome-scale protein function classification. Bioinformatics. 2014;30:1236-40.

45. Thomas PD, Campbell MJ, Kejariwal A, Mi H, Karlak B, Daverman R, et al. PANTHER: a library of protein families and subfamilies indexed by function Genome Res. 2003;13:2129-41.

46. Mi H, Muruganujan A, Casagrande JT, Thomas PD. Large-scale gene function analysis with the PANTHER classification system. Nat Protoc. 2013;8:1551-66.

47. Li H, Durbin R. Fast and accurate long-read alignment with BurrowsWheeler transform. Bioinformatics. 2010;26:589-95.

48. Li H, Handsaker B, Wysoker A, Fennell T, Ruan J, Homer N, et al. The Sequence Alignment/Map format and SAMtools. Bioinformatics. 2009;25:2078-9.

49. Robinson MD, McCarthy DJ, Smyth GK. edgeR: a Bioconductor package for differential expression analysis of digital gene expression data. Bioinformatics. 2010;26:139-40.
50. Love MI, Huber W, Anders S. Moderated estimation of fold change and dispersion for RNA-seq data with DESeq2. Genome Biol. 2014;15:550.

51. http://www.R-project.org/ (2016). Accessed 6 Jan 2016.

52. Livneh E, Glazer L, Segal D, Schlessinger J, Shilo BZ. The Drosophila EGF receptor gene homolog: conservation of both hormone binding and kinase domains. Cell. 1985:40:599-607.

53. Aroian RV, Koga M, Mendel JE, Ohshima Y, Sternberg PW. The let-23 gene necessary for Caenorhabditis elegans vulval induction encodes a tyrosine kinase of the EGF receptor subfamily. Nature. 1990;348:693-9.

54. Raz E, Schejter ED, Shilo BZ. Interallelic complementation among DER/flb alleles: implications for the mechanism of signal transduction by receptortyrosine kinases. Genetics. 1991;129:191-201.

55. Thacker C, Peters K, Srayko M, Rose AM. The bli-4 locus of Caenorhabditis elegans encodes structurally distinct kex2/subtilisin-like endoproteases essential for early development and adult morphology. Genes Dev. 1995;9:956-71.

56. Andrade MA, Perez-Iratxeta C, Ponting CP. Protein repeats: structures, functions, and evolution. J Struct Biol. 2001;134:117-31.

57. Kanai F, Liu H, Field SJ, Akbary H, Matsuo T, Brown GE, et al. The PX domains of p47phox and p40phox bind to lipid products of PI(3)K. Nat Cell Biol. 2001;3:675-8

58. Song X, Xu W, Zhang A, Huang G, Liang X, Virbasius JV, et al. Phox homology domains specifically bind phosphatidylinositol phosphates. Biochemistry. 2001;40:8940-4.

59. Wang Y, Oh SW, Deplancke B, Luo J, Walhout AJ, Tissenbaum HA. Caenorhabditis elegans 14-3-3 proteins regulate life span and interact with SIR-2.1 and DAF-16/FOXO. Mech Ageing Dev. 2006;127:741-7.

60. Li J, Tewari M, Vidal M, Lee SS. The 14-3-3 protein FTT-2 regulates DAF-16 in Caenorhabditis elegans. Dev Biol. 2007;301:82-91.

61. Duret L, Guex N, Peitsch MC, Bairoch A. New insulin-like proteins with atypical disulfide bond pattern characterized in Caenorhabditis elegans by comparative sequence analysis and homology modeling. Genome Res. 1998;8:348-53.

62. Kawano T, Ito Y, Ishiguro M, Takuwa K, Nakajima T, Kimura Y. Molecular cloning and characterization of a new insulin/IGF-like peptide of the nematode Caenorhabditis elegans. Biochem Biophys Res Commun. 2000;273:431-6

63. Pierce SB, Costa M, Wisotzkey R, Devadhar S, Homburger SA, Buchman AR, et al. Regulation of DAF-2 receptor signaling by human insulin and ins-1, a member of the unusually large and diverse $C$. elegans insulin gene family. Genes Dev. 2001;15:672-86.

64. Li W, Kennedy SG, Ruvkun G. daf-28 encodes a C. elegans insulin superfamily member that is regulated by environmental cues and acts in the DAF-2 signaling pathway. Genes Dev. 2003;17:844-58.

65. Stoltzfus JD, Minot S, Berriman M, Nolan TJ, Lok JB. RNAseq analysis of the parasitic nematode Strongyloides stercoralis reveals divergent regulation of canonical dauer pathways. PLoS Negl Trop Dis. 2012;6:e1854.

66. Murphy CT, Lee SJ, Kenyon C. Tissue entrainment by feedback regulation of insulin gene expression in the endoderm of Caenorhabditis elegans. Proc Natl Acad Sci USA. 2007;104:19046-50.

67. Cornils A, Gloeck M, Chen Z, Zhang Y, Alcedo J. Specific insulin-like peptides encode sensory information to regulate distinct developmental processes. Development. 2011:138:1183-93.

68. Chen Z, Hendricks M, Cornils A, Maier W, Alcedo J, Zhang Y. Two insulin-like peptides antagonistically regulate aversive olfactory learning in C. elegans. Neuron. 2013;77:572-85

69. Hristova M, Birse D, Hong Y, Ambros V. The Caenorhabditis elegans heterochronic regulator $\mathrm{LIN}-14$ is a novel transcription factor that controls the developmental timing of transcription from the insulin/insulin-like growth factor gene ins-33 by direct DNA binding. Mol Cell Biol. 2005;25:11059-72

70. Baugh LR, Kurhanewicz N, Sternberg PW. Sensitive and precise quantification of insulin-like mRNA expression in Caenorhabditis elegans. PLoS One. 2011;6:e18086.

71. Stoltzfus JD, Bart SM, Lok JB. CGMP and NHR signaling co-regulate expression of insulin-like peptides and developmental activation of infective larvae in Strongyloides stercoralis. PLoS Pathog. 2014;10:e1004235.

72. Song MS, Salmena L, Pandolfi PP. The functions and regulation of the PTEN tumour suppressor. Nat Rev Mol Cell Biol. 2012;13:283-96.

73. Hollander MC, Blumenthal GM, Dennis PA. PTEN loss in the continuum of common cancers, rare syndromes and mouse models. Nat Rev Cancer. 2011;11:289-301. 
74. Oh SW, Mukhopadhyay A, Svrzikapa N, Jiang F, Davis RJ, Tissenbaum HA JNK regulates lifespan in Caenorhabditis elegans by modulating nuclear translocation of forkhead transcription factor/DAF-16. Proc Natl Acad Sci USA. 2005;102:4494-9.

75. Ayyadevara S, Alla R, Thaden JJ, Shmookler Reis RJ. Remarkable longevity and stress resistance of nematode PI3K-null mutants. Aging Cell. 2008;7:13-22.

76. Alam H, Williams TW, Dumas KJ, Guo C, Yoshina S, Mitani S, et al. EAK-7 controls development and life span by regulating nuclear DAF-16/FoxO activity. Cell Metab. 2010;12:30-41.

77. Hertweck M, Göbel C, Baumeister R. C. elegans SGK-1 is the critical component in the Akt/PKB kinase complex to control stress response and life span. Dev Cell. 2004;6:577-88

78. Karathanassis D, Stahelin RV, Bravo J, Perisic O, Pacold CM, Cho W, et al. Binding of the PX domain of p47(phox) to phosphatidylinositol 3,4bisphosphate and phosphatidic acid is masked by an intramolecular interaction. EMBO J. 2002;21:5057-68.

79. Accili D, Arden KC. FoxOs at the crossroads of cellular metabolism, differentiation, and transformation. Cell. 2004;117:421-6.

80. Lee RY, Hench J, Ruvkun G. Regulation of C. elegans DAF-16 and its human ortholog FKHRL1 by the daf-2 insulin-like signaling pathway. Curr Biol. 2001;11:1950-7.

81. Lin K, Hsin H, Libina N, Kenyon C. Regulation of the Caenorhabditis elegans longevity protein DAF-16 by insulin/IGF-1 and germline signaling. Nat Genet. 2001;28:139-45.

82. Henderson ST, Johnson TE. daf-16 integrates developmental and environmental inputs to mediate aging in the nematode Caenorhabditis elegans. Curr Biol. 2001;11:1975-80.

83. Kwon ES, Narasimhan SD, Yen K, Tissenbaum HA. A new DAF-16 isoform regulates longevity. Nature. 2010;466:498-502.

84. Massey Jr HC, Nishi M, Chaudhary K, Pakpour N, Lok JB. Structure and developmental expression of Strongyloides stercoralis fktf-1, a proposed ortholog of daf-16 in Caenorhabditis elegans. Int J Parasitol. 2003;33:1537-44.

85. Brand A, Hawdon JM. Phosphoinositide-3-OH-kinase inhibitor LY294002 prevents activation of Ancylostoma caninum and Ancylostoma ceylanicum third-stage infective larvae. Int J Parasitol. 2004;34:909-14.

86. Huang SC, Chan DT, Smyth DJ, Ball G, Gounaris K, Selkirk ME. Activation of Nippostrongylus brasiliensis infective larvae is regulated by a pathway distinct from the hookworm Ancylostoma caninum. Int J Parasitol. 2010;40:1619-28.

87. Stoltzfus JD, Massey Jr HC, Nolan TJ, Griffith SD, Lok JB. Strongyloides stercoralis age-1: a potential regulator of infective larval development in a parasitic nematode. PLoS One. 2012;7:e38587.

88. Gibbs HC. Hypobiosis in parasitic nematodes - an update. Adv Parasitol. 1986;25:129-74

\section{Submit your next manuscript to BioMed Central and we will help you at every step:}

- We accept pre-submission inquiries

- Our selector tool helps you to find the most relevant journal

- We provide round the clock customer support

- Convenient online submission

- Thorough peer review

- Inclusion in PubMed and all major indexing services

- Maximum visibility for your research

Submit your manuscript at www.biomedcentral.com/submit 